\title{
Detection of Incompetent Perforating Veins by Venography at Operation
}

\author{
JULIAN TOWNSEND,* M.B., B.CHIR., F.R.C.S.; HUGH JONES, $\dagger$ M.S., F.R.C.S. \\ J. EDMUND WILLIAMS, $\ddagger$ B.SC., M.R.C.P.ED., F.F.R.
}

Brit. med.F., 1967, 3, 583-585

All types of varicose veins can be treated successfully by a radical stripping operation in which the whole system of diseased veins and related perforating veins is removed (Myers, 1957 ; Arenander, 1960 ; Sherman, 1964). Simple procedures such as high ligation, with or without stripping of the main saphenous trunk, though widely practised, are much less successful, even in the early and uncomplicated case. Great saphenous incompetence is usually obvious, and can be convincingly demonstrated by the Brodie-Trendelenburg test. Femoro-saphenous reflux fills the superficial veins with blood at a low pressure only (Cockett, 1956).

Incompetent perforating veins, however, are easy to overlook, and their presence cannot be excluded with certainty by any clinical test. They are present in $90 \%$ of cases (Sherman, 1949), and are especially important as a cause of varices which persist or recur after operation (Mathiesen, 1953 ; Lofgren, Myers and Webb, 1956 ; and many others), and as the cause of ulcers. Cockett and Jones (1953) showed that the venous drainage of the lower part of the leg is through short perforating veins to the deep vessels, and only indirectly through the saphenous systems. Thus in this area any superficial venous disease is likely to stem from the perforating vessels, and it is here in the "gaiter-area" that the high intermittent pressures in the deep veins can be transmitted to the surface through incompetent perforating veins, leading to ulceration (Arnoldi, 1966). "Varicose ulceration" due solely to incompetence of superficial veins does not occur, for incompetent perforating veins can be found in association with every venous ulcer (Sarjeant, 1964 ; Arnoldi, 1966 ; Haeger, 1966). It is true that saphenous incompetence may be a contributory cause of ulceration and that ulcers may be healed by removal of the saphenous vein, but the effect is unpredictable and healing often only temporary (Moore, 1957 ; Haeger, personal communication, 1966).

It is sometimes thought that stripping out the saphenous trunk destroys the perforating veins, but Sherman (1949) has shown that about one-half of all perforating veins, and most of the incompetent ones, are not directly connected with the saphenous trunks. Radical stripping operations, though repeatedly shown to be very effective, are not practised by the majority of surgeons, who perhaps feel that the time and trouble required and the trauma to the patient are not justified even by a reasonably certain cure. Another prevalent view, that varicosis is a progressive condition essentially unsuited to radical cure, has some basis in fact, but is founded more on a practice of inadequate diagnosis followed by inadequate surgery.

Preoperative diagnosis of incompetent perforating veins is difficult. Various clinical signs are described but none is reliable. Surgeons experienced in venous disease commonly find at operation incompetent perforating veins which they have not detected previously. The lesser operations which are commonly practised often leave incompetent perforating veins intact, with the attendant risk of persistent or recurrent varices and ulcers. If it were possible to locate incompetent perforating veins accurately and confidently it is probable that they would be

- Senior Surgical Registrar.

+ Consultant Surgeon.

₹ Consultant Surgeon.

Varicose Vein Clinic, Cardiff Royal Infirmary. ligated more frequently and with greater economy than is now usual, and with improved results. For this reason some methods of venography have been examined and the findings assessed by surgical exploration. A technique of venography has been evolved which with practice is easy to perform, easy to interpret, and reliable.

It is widely held that there is no place for routine venography in the treatment of varicose veins. This is not our experience. Accurate diagnosis leads to precise surgery, and may obviate the need for a formal operation, such as that of Cockett and Jones (1953), in which an incision half the length of the tibia is sometimes made in order to ligate a single incompetent perforating vein, or even without finding one at all (Dodd and Cockett, 1956).

The results of venography were compared with a number of common physical signs, the most useful of which was found to be palpation of fascial defects. We agree with many other writers that the fascial defect lies not in the deep fascia, where a hole is rarely palpable, but in the superficial fascia, where a varix lies at the superficial end of an incompetent perforating vein. All fascial defects and all incompetent perforating veins shown by venography were exposed at operation, and the competence of any perforating vein was tested by the bleed-back test of Turner Warwick (1931).

\section{Method}

Before operation the position of the dilated veins was marked on the skin. The leg was then palpated, and all fascial defects were marked, and then labelled with a lead shot secured by adhesive plaster.

The anaesthetized patient was placed on a radiolucent table top, with a 5-degree foot-down tilt. Under aseptic conditions a sternal-puncture needle was inserted horizontally deep into the lower end of the tibia in the position shown in Fig. 1. Injection of a little isotonic saline and the withdrawal of a stream of blood into the syringe confirmed that the needle was correctly placed. Difficulty in making the injection was due to the needle being either too low or not far enough in. With the leg in the anatomical position the $x$-ray tube and film were placed to take an anteroposterior view, with the ankle joint just included at the lower end.

The veins were drained by raising the leg to 30 degrees for 30 seconds. Omission of this step led to excessive dilution of the opaque medium. The leg was lowered and $20 \mathrm{ml}$. of warm $45 \%$ Hypaque (sodium diatrizoate) was injected in 30 seconds. As soon as injection was completed the foot was replaced in the anatomical position and the film exposed. A second film was placed to include the femoral condyles and exposed immediately after gently flexing the foot up and down. The leg was again raised to ensure that no medium remained stagnant in the veins, and any possible risk of thrombosis was avoided.

\section{Interpretation}

Incompetence is almost certain when a perforating vein is seen with localized filling of veins at its superficial end, for then 
flow must have been outward (Fig. 2, a, b, and c). When several incompetent perforating veins are present there may be dense filling of some superficial veins, and it is then impossible to say whether a given perforator has filled from within or without. An abnormal perforator is enlarged and may be irregular or tortuous superficially ; there may be localized dilatation of the deep or superficial veins at either end of it. A localized superficial varix is a strong indication of the presence of an incompetent perforating vein (Fig. 3, b).

Any medial perforator which fills from within is incompetent, but lateral muscular perforators, which are often large and double, may fill from within yet be competent unless there is also definite filling of overlying superficial veins (Fig. 2, c and $\mathrm{d}$ ).

When an incompetent perforating vein has been recognized on the $x$-ray film it is not always easy to find the corresponding site on the leg, but by reference to the lead markers it is possible to gauge the level with great accuracy. This information, in conjunction with the skin-markings of varices and fascial defects, is interpreted with reference to the anatomy of the perforating veins, good accounts of which have been given by Sherman (1949) and Sarjeant (1964), and usually allows accurate location of the site of incompetence.

When the radiographic information is considered on its own it is likely to be less valuable than when combined with clinical signs. For this reason it is advised that the surgeon should co-operate in the interpretation of the venograms, especially as he has the opportunity to examine the veins at operation, and so correlate the clinical and radiographic signs with the actual state of the veins.

Anteroposterior views only were used, and proved adequate, firstly because nearly all the clinically important perforating veins lie in the tibial and peroneal groups, which show best in this projection, and secondly because the incompetent posterior muscular perforating veins are easy to recognize clinically.

The method of intraosseous venography does not invariably show the deep veins, and reliable information on these can only be obtained by other techniques.

\section{Results}

So far 31 patients have been treated and 46 legs operated on. It is believed that few incompetent perforating veins remained undiscovered, because during the course of the operations many perforating veins were seen by chance at sites other than those under investigation, and all these additional perforating veins appeared normal. Those tested by the method of Turner Warwick (1931) were competent.

The clinical, venographic, and operative findings are shown in the Table.

Results of Exploration at All Sites Where Incompetent Perforating Veins (I.P.V.) Were Diagnosed by Clinical Signs and by Venography

\begin{tabular}{|c|c|c|c|c|}
\hline \multicolumn{2}{|c|}{ Preoperative Diagnosis } & \multicolumn{3}{|c|}{ Findings on Exploration } \\
\hline $\begin{array}{l}\text { Diagnostic Procedure } \\
\text { Leading to Exploration }\end{array}$ & No. of Sites & $\begin{array}{l}\text { Diagnosis } \\
\text { Confirmed } \\
\text { (I.P.V. } \\
\text { Found) }\end{array}$ & $\begin{array}{c}\text { Diagnosis } \\
\text { not } \\
\text { Confirmed } \\
\text { (No I.P.V. } \\
\text { Found) }\end{array}$ & $\begin{array}{c}\text { I.P.V. } \\
\text { Found } \\
\text { Elsewhere }\end{array}$ \\
\hline $\begin{array}{lll}\text { Clinical signs } & \ldots & \ldots \\
\text { Venography } & \ldots & \ldots \\
\end{array}$ & $\begin{array}{l}112 \\
109\end{array}$ & $\begin{array}{l}72 \\
96\end{array}$ & $\begin{array}{l}40 \\
13\end{array}$ & $\begin{array}{r}32 \\
8\end{array}$ \\
\hline $\begin{array}{l}\text { Total (clinical signs, } \\
\text { venography, or both) }\end{array}$ & 156 & 104 & 52 & Nil \\
\hline
\end{tabular}



FIG. 1.-The needle is inserted iust above the base of the medial (A Salah needle, gauge 15 or 12 , was used for most of these venograms, but several other types were used.)

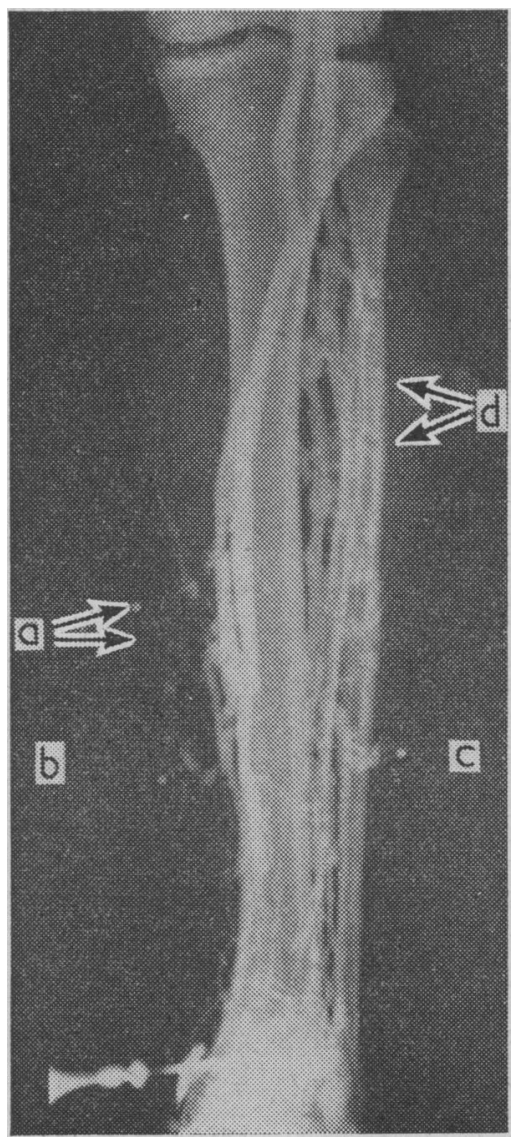

Fig. 2.-a, b, and c, Incompetent perforating veins with filling of overlying superficial veins. d, Paired peroneal muscular veins filling from the deep veins. Note the paired deep calf veins (normal) and paired popliteal veins (variation).

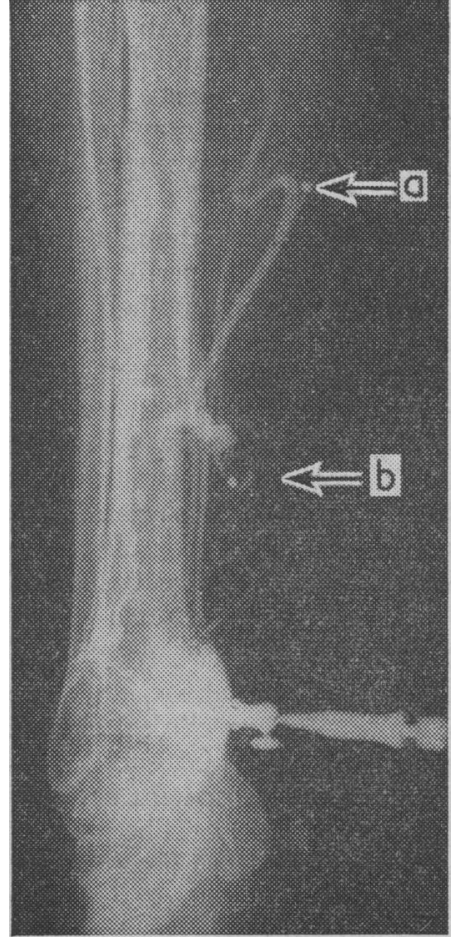

FIG. 3.-a, Fascial defect (with lead marker) seen to be due to superficial varix only. b, Incompetent perforating vein with overlying superficial varix. Note the distance between the fascial defect and the perforating vein. 
A total of 104 incompetent perforating veins were found at operation. Clinical signs had detected $72(69 \%)$ of these and missed $32(31 \%)$. A further 40 explorations were made on clinical grounds without any incompetent perforating vein being found. Venograms had detected $96(92 \%)$ of the 104 incompetent perforating veins, but had failed to detect the remaining eight. Another 13 sites were explored because of venographic signs, but no incompetent perforating veins were found.

The distribution of all the incompetent perforating veins found is shown in Fig. 4 and corresponds well with that found by Sherman (1949) in nearly a thousand radical operations.

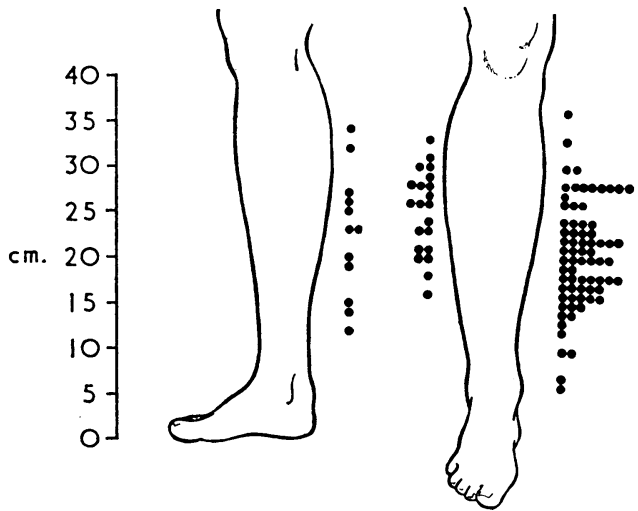

FIG. 4.-Distribution of incompetent perforating veins in 46 legs with varices. The medial group are those lying behind the those lying behind the tibia, the lateral group are closely related ro the fibula. The remainder were posterior muscular perforating veins; all of these were detected clinically, but three were not detected by venography. There were no incompetent anterior perforating veins in this group of patients.

\section{Discussion}

Intraosseous venograms are easy to interpret because of the lack of superficial venous filling (Fig. 5). We have found the calcaneum a little less satisfactory in this respect than the malleoli. We have also used the lateral malleolus, as recommended by Cockett (1953), but have found that demonstration of the important perforating veins medial to the lower third of the tibia was not always reliable. Injection into the lower tibia has proved better: it is a little more difficult to insert the needle, but satisfactory injection can always be made and films of diagnostic value obtained.

The method described is simple to perform and requires few extra facilities. The whole examination should add less than 10 minutes to the operating-time for bilateral varices. There have been no complications. The precise information obtained is of value in two ways: firstly, the operation can be more comprehensive than when reliance is placed on clinical tests alone, and, secondly, the operation can be planned to minimize the number and extent of the incisions. The technique and interpretation are likely to give difficulty if venography is used only as an occasional procedure to investigate the complex problem, but they become simple with practice.

Even in "primary" long saphenous varices an incompetent perforating vein is of ten present and may be surprisingly large. Such a vein can easily be ligated through a small incision over it. A variety of cases have been treated, ranging from simple "primary" varices to post-thrombotic ulcers. Venography proved helpful in 36 of the 46 legs and in all types of case. Despite the fact that excision of varices was not practised, very few postoperative injections have been required.

\section{Summary}

Incompetent perforating veins are usually present with varicose veins. They are responsible for most of the complications and for some recurrences. They should be obliterated.

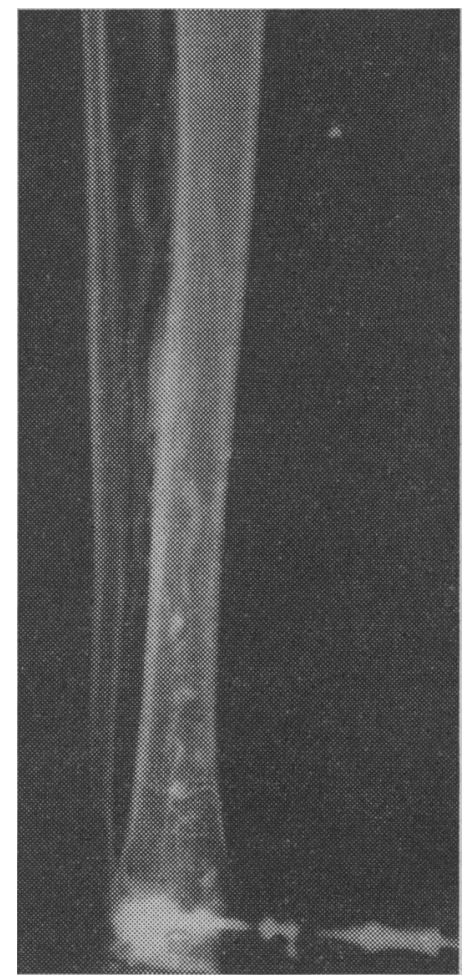

FIG. 5.-No incompetent perforating veins present. The dye passes from the bone marrow to dhe passes from but none escapes into the superficial veins.

A method of operative venography is described which has proved reliable in the detection of incompetent perforating veins and much superior to clinical examination alone.

The information obtained made possible a more comprehensive and more precise operation in three out of every four legs treated.

We thank the radiographers of Cardiff Royal Infirmary and Llandough Hospital for their willing assistance, and Miss Kathleen Davies for patient secretarial help.

Requests for reprints should be addressed to Mr. J. Townsend, c/o the Secretary, Varicose Vein Clinic, Royal Infirmary, Cardiff.

\section{REFERENCES}

Arenander, E. (1960). Acta chir. scand., Suppl. No. 260.

Arnoldi, C. C. (1966). Ibid., 132, 6?8.

Cockett, F. B. (1953). Brit. F. Radio!., 26, 339

(1956). Brit. med. F., 2, 1399.

- and Jones, D. E. E. (1953). Lancet, 1, 17

Dodd, H., and Cockett, F. B. (1956). The Pathology and Surgery of the Veins of the Lower Limb, p. 358 . London.

Haeger, K. (1966). Venous and Lymphatic Disorders of the Leg, p. 86. Copenhagen.

Lofgren, K. A.. Myers, T. T., and Webb, W. D. (1956). Surg. Gynec. Obstet., 102, 729.

Mathiesen, F. R. (1953). Acta chir. scand., 105, 376.

Moore, J. M. (1957). Scot. med. 7., 2, 319.

Myers, T. T. (1957). F. Amer. med. Ass., 163, 87.

Sarjeant, T. R. (1964). Surg. Clin. N. Amer., 44, 1383.

Sherman, R. S. (1949). Ann. Surg., 130, 218.

- (1964). Surg. Clin. N. Amer., 44, 1369.

Warwick, W. T. (1931). The Rational Treatment of Varicose Veins and Varicocele, p. 60 . London 